\title{
NuEra tight: efficacy and safety in the treatment of the appearance of cellulite with minimal downtime
}

\author{
Sergey Y Turin, $\mathrm{MD}^{1} \mathbb{1}$, Christopher J Micallef, $\mathrm{DO}^{2} \mathbb{1}$, Clark F Schierle, MD, PhD ${ }^{1}$ \\ 'Division of Plastic and Reconstructive Surgery, Northwestern Feinberg School of Medicine, Chicago, IL, United States, \\ ${ }^{2}$ Division of Plastic and Reconstructive Surgery, University of Illinois at Chicago School of Medicine, Chicago, IL, United States
}

\begin{abstract}
Background: Cellulite is a complex aesthetic problem caused by a complex interplay of dermal-fascial septae, lipodystrophy, and inadequate skin elasticity, but reliable solutions for this condition remain elusive.

Objective: To evaluate a novel radiofrequency-based body contouring device cleared by the FDA for the temporary improvement in the appearance of cellulite in the thighs and abdomen (NuEra; Lumenis, Ltd., Yokneam, Israel).

Methods: A prospective cohort trial was designed to first collect subjective patient and provider reported outcome measures of safety, comfort, and efficacy of the NuEra system in treatment of thigh cellulite in 9 participants. Following review of this data, the second portion of the trial was conducted using the NuEra system to treat the abdominal area in the same patients, collecting anthropometric measurements using a 3-dimensional imaging system (Vectra M3; Canfield, Parsippany, NJ, USA). Treatment protocol specified 5 treatments at approximately 1 week intervals with a final treatment after a 6 week delay. Results were assessed at the time of the treatments and at two month following the last treatment. Likert scales were used to evaluate subjective provider and patient experience and a standardized cellulite assessment scale was used to rate the pre and post treatment photographs.

Results: Patients rated the overall level of comfort at 4.3 and satisfaction as 3.3 on a 5-point scale. Pain scale scores during treatment averaged 1.4 on a 5-point scale. Providers reported very high levels of satisfaction with the ease of the treatment (4.3 on a 5 -point scale). Evaluation of standardized photographs showed an average improvement of 0.53 (standard deviation [SD] $=0.53$ ), with the average pre-treatment score of $2.2(S D=0.62)$ and post-treatment score of $1.64(S D=0.68)$. The average reduction in waist and hip circumference measurements was $0.99 \mathrm{~cm}(\mathrm{SD}=2.68 \mathrm{~cm})$ and $0.71 \mathrm{~cm}(\mathrm{SD}=1.44 \mathrm{~cm})$.

Conclusion: Radiofrequency based body contouring is a safe, effective, and well tolerated treatment for thigh cellulite.
\end{abstract}

Keywords: body contouring; cellulite; noninvasive; radiofrequency; skin texture

\section{Introduction}

Cellulite refers to the dimpled or lumpy appearance of the skin caused by adipose tissue that is displaced from its normally deep location to the more superficial layer of connective tissue just beneath the skin. It is primarily found in the lower body (buttocks, hips, and thighs), affects $80 \%$ to $90 \%$ of post pubertal females, and has been associated with age as well as a number of hormonal, hereditary, and lifestyle factors. The appearance of cellulite is one of the true unsolved problems in aesthetics today [1], and growing demand from patients and physicians for effective treatment options has emphasized the lack of satisfaction with the current cellulite reduction methodologies [2-4].

Previous generations of energy-based therapies have shown

Received November 25, 2020; Revised December 10, 2020; Accepted December 12, 2020

Corresponding author: Sergey Y Turin

E-mail: Sergey.Turin@northwestern.edu

This is an Open Access article distributed under the terms of the Creative Commons Attribution Non-Commercial License (http://creativecommons.org/licenses/by-nc/4.0), which permits unrestricted non-commercial use, distribution, and reproduction in any medium, provided the original work is properly cited.

Copyright ( 2020 Korean Society of Korean Cosmetic Surgery and Medicine (KSKCS \& KCCS). 
promise in treating cellulite [3] and the NuEra Tight (Lumenis, Ltd., Yokneam, Israel) device is the latest entrant to this field. This device targets the subcutaneous tissues thought to be culpable for the appearance of cellulite by delivering radiofrequency (RF) energy at $470 \mathrm{kHz}$. NuEra Tight is currently FDA-cleared for RF heating of the skin and immediately underlying tissues, both superficial and deep, to treat a number of conditions, including the temporary reduction in the appearance of cellulite.

The authors herein present their initial experience in the application of NuEra Tight for thigh and abdomen cellulite treatment in the clinical setting.

\section{Materials and methods}

A prospective case series study was designed following the Guidelines of the Department of Health and Human Services (DHHS) Regulations for the Protection of Human Subjects, to evaluate the safety and efficacy of the intervention. Subject inclusion criteria were female sex, age between 18 and 65 years, subjective dissatisfaction with appearance of their upper leg/ thigh region, and objective rating of mild or greater $(>1)$ on the Cellulite Severity Scale [5] by the provider. Exclusion criteria were inability to complete the entire treatment series, loss to follow-up before the final result photographs were obtained, or undergoing any other treatment of the skin or subcutaneous tissues of the treated areas that could be deemed to confound the study results.

Nine consecutive subjects were enrolled between December 2018 and April 2019. After obtaining consent for data and photography collection, the subjects underwent treatment of thigh and abdomen cellulite by the NuEra Tight device in the senior author's (CFS) practice.

Every participant underwent 6 treatments to each area (thighs and abdomen), with the first five treatments administered at intervals of 7-10 days, with the sixth treatment administered at the time of the last follow-up (six weeks after the fifth treatment), such that the entire course spanned 11-13 weeks. Treatments were delivered by 4 providers. The device was used in assisted mode, set for "Thigh Cellulite" to protocol the treatment to $40^{\circ} \mathrm{C}$ tissue temperature endpoint for 20 minutes, delivered via the $80 \mathrm{~mm}$ mono polar handpiece. The cellulite treatment protocol requires that the area treated in a single session not cover more than 4 times the area of the handpiece electrode size, necessitating splitting up the treatments in certain patients with a larger target surface area was. Participants were photographed at baseline, before treatments five and six, and then at one week and one month after the last (sixth) treatment. Three dimensional (3D) photography was also obtained at these timepoints using the office 3D imaging system (Vectra M3; Canfield, Parsippany, NJ, USA).

Photographs of the participants prior to any treatment and at the four week follow up were rated by two of the authors (SYT and CJM) and the appearance of cellulite in the thigh area was rated on the Cellulite Dimples-At Rest scale, as per Hexsel at al [5]. Anthropometric data from the 3D photographs was analyzed to evaluate changes in abdominal and hip circumference measurements, according to established protocols [6]. The abdominal circumference measurement was centered at the level of the umbilicus and the hip circumference measurement was measured at $10 \mathrm{~cm}$ below the level of the umbilicus.

As part of routine practice, patients were asked to rate their treatments on a $0-10$ scale for comfort (with 0 representing maximal discomfort/pain and 10 being maximal comfort). Patients rated their treatment for whether it was beneficial and met their expectations on a 1 to 5 scale (with 1 being "strongly disagree" and 5 being "strongly agree"). Satisfaction with the treatment results was also rated on a 1 to 5 scale (with 1 being "not improved" and 5 being "very much improved"). Patients also rated whether they would recommend NuEra Tight to family and friends on a 1 to 5 scale (with 1 being "unlikely to recommend" and 5 being "very much likely to recommend"). Providers were also asked to rate the treatments on a 1-to-10 scale for ease of use, pain at onset, pain at peak, pain at plateau, and time of peak (in minutes). The limited sample size of this pilot study would preclude calculations for statistical significance from being clinically meaningful, so these were omitted for the time being.

\section{Ethical approval}

All procedures performed in studies involving human participants were in accordance with the ethical standards of the institutional and/or national research committee and with the 1964 Helsinki declaration and its later amendments or comparable ethical standards.

\section{Results}

Nine participants were enrolled in the study and underwent all six treatments per the protocol. Average participant age was 37.3 years (standard deviation $[\mathrm{SD}]=12.2$ years) and average BMI was $21.3 \mathrm{~kg} / \mathrm{m}^{2}\left(\mathrm{SD}=2.0 \mathrm{~kg} / \mathrm{m}^{2}\right)$. 


\section{Patients-rated parameters}

Patients rated NuEra Tight highly on the measure of comfort, with an average of $4.3(\mathrm{SD}=0.7)$. Patients were moderately satisfied with the overall results of the procedure, with an average rating of $3.3(\mathrm{SD}=1.2)$. Patients stated that the procedure generally met their expectations, with an average $3.1(\mathrm{SD}=1.2)$ rating. Patients were quite willing to recommend this procedure to others, rating that an average of $2.7(\mathrm{SD}=1.6)$. They rated the benefits of their procedure an average of $3.3(\mathrm{SD}=1.2)$.

\section{Photographic rating}

The average change on the Cellulite-At Rest scale [5] was
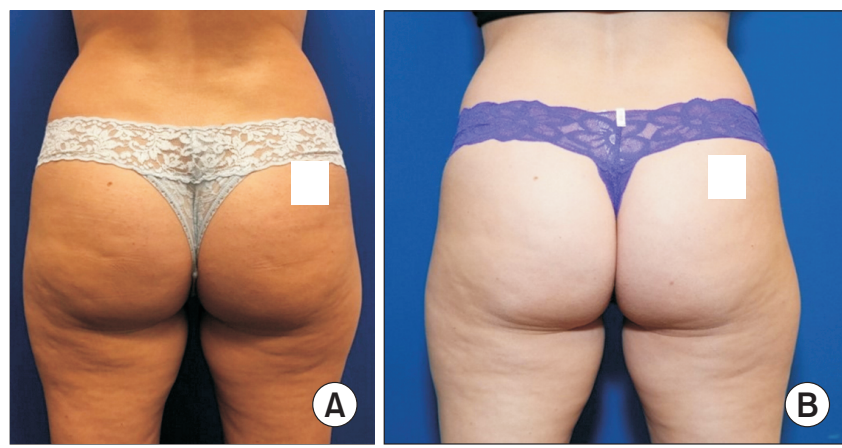

Fig. 1. Patient A. (A) Pre-treatment appearance; (B) appearance after 6 treatments at 1 month follow-up after the last treatment. This image has been modified (white rectangle) to conceal identifying features.
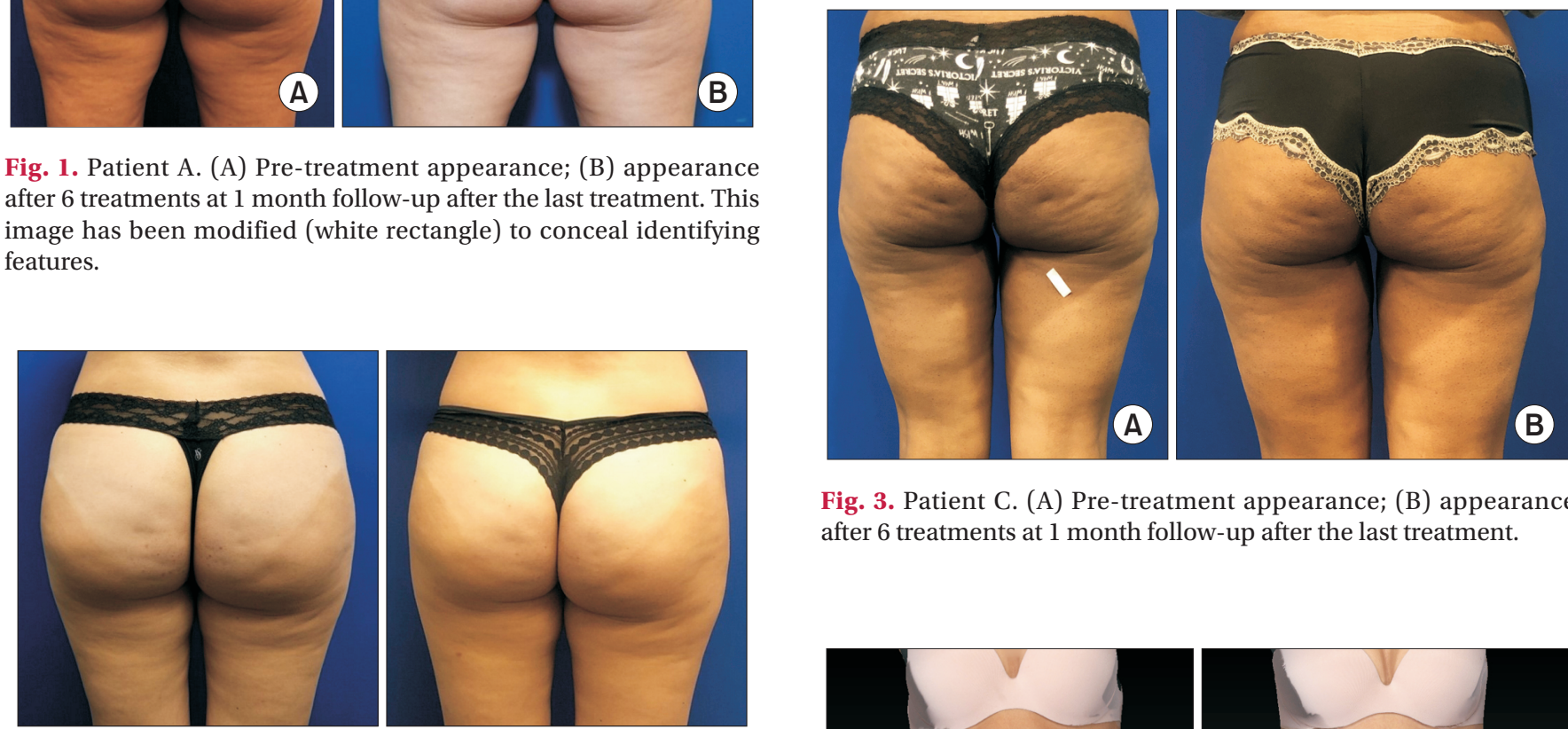

Fig. 3. Patient C. (A) Pre-treatment appearance; (B) appearance after 6 treatments at 1 month follow-up after the last treatment.
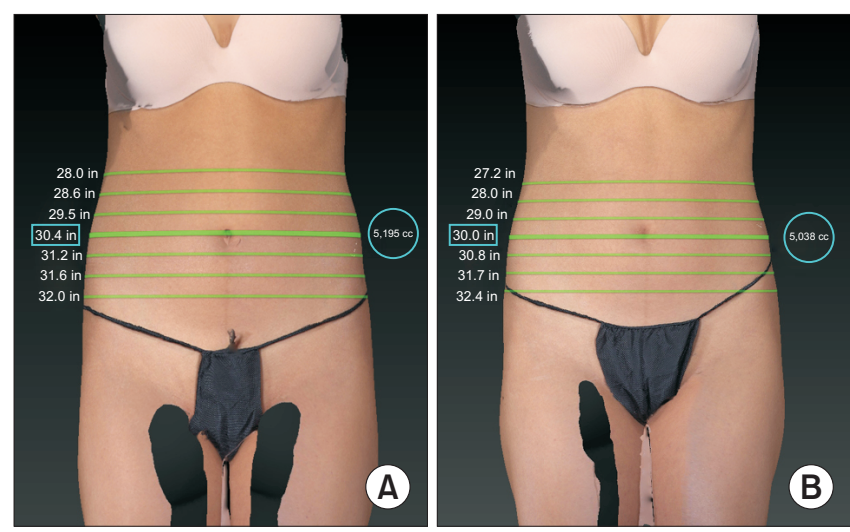

Fig. 2. Patient B. (A) Pre-treatment appearance; (B) appearance after 6 treatments at 1 month follow-up after the last treatment.

$-0.53(\mathrm{SD}=0.53)$, with the average pre-treatment score of 2.2 $(\mathrm{SD}=0.62)$ and post-treatment score of 1.64 ( $\mathrm{SD}=0.68)$. Representative results are shown in Fig. 1-3.

\section{Provider-rated parameters}

Providers found the NuEra Tight very easy to use and administer, rating it an average $8.5(\mathrm{SD}=1.0)$ on a $0-10$ scale regarding ease of use. During the procedure administration, provider perception of pain at onset was low, with an average 1.0 rating. Pain increases at the peak of the procedure, with an average 6.5 $(\mathrm{SD}=1.3)$ rating from providers, but plateaus at a low level of 2.8 $(\mathrm{SD}=1.0)$. The time it takes to reach the peak is relatively low, at an average of 2.3 minutes ( $\mathrm{SD}=0.5$ minutes).

\section{Waist and hip circumference measurement}

The average pre-treatment waist and hip circumference measurements were $74.79 \mathrm{~cm}(\mathrm{SD}=5.25 \mathrm{~cm})$ and $86.22 \mathrm{~cm}(\mathrm{SD}=7.89$

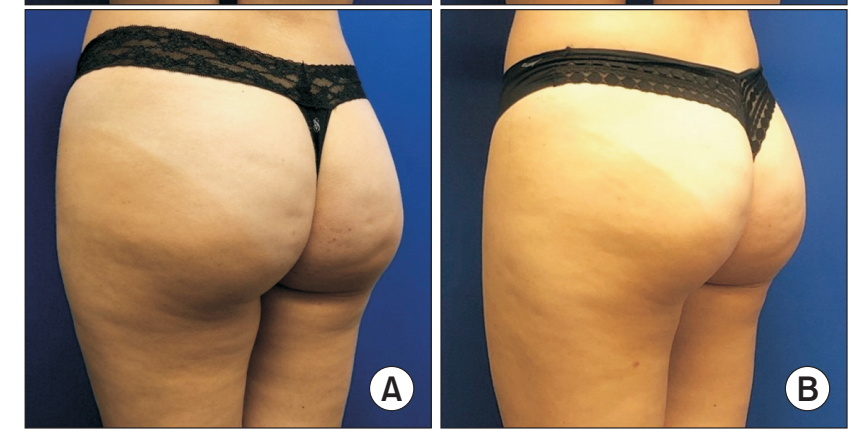

Fig. 4. Example for pre-treatment (A) and post-treatment (B) 3-dimensional measurements of abdominal and hip circumference. 
$\mathrm{cm})$, respectively. Average waist and hip circumference measurements at 1 month follow-up after the last treatment were $73.8 \mathrm{~cm}(\mathrm{SD}=6.52 \mathrm{~cm})$ and $85.51 \mathrm{~cm}(\mathrm{SD}=7.49 \mathrm{~cm})$, respectively. Average reduction in waist and hip circumference measurements was $0.99 \mathrm{~cm}(\mathrm{SD}=2.68 \mathrm{~cm})$ and $0.71 \mathrm{~cm}(\mathrm{SD}=1.44 \mathrm{~cm})$. Representative measurements are shown in Fig. 4.

\section{Discussion}

Despite the prevalence of cellulite and the large amount of resources dedicated to it, efforts to produce an effective medical or device-based intervention for the appearance of cellulite are still ongoing. The thigh area is notoriously difficult to treat due to the interaction of skin tethering ligaments, fat distribution in that area, and often, poor skin elasticity. The difficulty of the challenge is highlighted by the lack of efficacy for even more invasive modalities in the reduction of thigh cellulite.

Demand for non-surgical options also continues to grow as patients are becoming more aware of low-downtime treatment options [7], creating a niche for an expanding portfolio of devices developed to ameliorate the undesirable appearance of cellulite with minimal recovery. Naturally, RF based technologies have found a natural application in this arena. Initially used for skin tightening in the lower face and neck, RF devices utilize multiple electrodes (poles) to deliver energy to the dermis and create a controlled thermal injury. The resulting collagen denaturation and natural scar healing process that follow (particularly the fibroblast activity) results in tightening of the treated area's dermis, analogous to a controlled skin contracture in the setting of a burn [8], and the efficacy of RF for skin rejuvenation is supported in a number of studies [9]. Treating cellulite using RF relies on the same principles, but shifts the focus to the deeper, subdermal adipose and connective, such that the resulting metabolic response and wound healing yield an improvement in skin texture [10]. This is a newer application of this modality, but the body of literature supporting the use of RF for treating cellulite is growing rapidly $[11,12]$. Treatment with $\mathrm{RF}$ devices has been reported to effect up to a $3.75 \%$ reduction in abdominal circumference at 4-weeks post-treatment, with improvement in the appearance of cellulite and striae distensae [13].

The currently available RF devices differ in the method of thermal energy delivery by varying the electrode configuration. The most popular designs are mono polar and bipolar handsets. The principle of action for these arrangements is not dissimilar to the commonly used mono polar and bipolar elec- trosurgery devices. The mono polar devices use one negatively charged active electrode and one positively charged grounded electrode that is remote to the treatment area, forcing a wider and deeper distribution of the delivered energy. On the other hand, bipolar RF devices use one negative electrode and one positive electrode that are both in contact with the skin in the treatment area, creating a more direct energy delivery pathway - this is thought to be more efficacious for treating superficial tissues [14].

The NuEra Tight device emits RF waves at a frequency of 470 $\mathrm{kHz}$, delivering up to $250 \mathrm{~W}$ of energy to a specified depth, using either a unipolar or a bipolar handpiece. To limit overtreatment, the device is equipped with the APIC (Automatic Power and Impedance Control) system, which uses impedance detection to titrates the RF power delivered to the tissues so that the target temperature is maintained for the duration of the treatment. The automatic and gradual energy delivery modulation is a key component in ensuring consistent results and a maximal safety margin.

Among the current options for treating thigh and abdominal cellulite, NuEra Tight has shown great promise in managing this difficult therapeutic area. As our results demonstrate, it is subjectively highly rated by providers (average 8.5 out of 10) for ease of use and comfort by patient (average 4.3 out of 5). Moreover, we showed a significant objective average improvement of 0.53 points on the $0-4$ Cellulite scale as well as a $0.99 \mathrm{~cm}$ and 0.71 $\mathrm{cm}$ average decreased in waist and hip circumference measurements. The high comfort rating and speed of the procedure can be an important decision factor for patients wishing to avoid the discomfort of more invasive procedures or surgery. In addition, our results revealed high provider satisfaction with the system due to ease of use and administration in terms of practical use.

The results presented above, both subjective and photographic, support the benefit of RF energy, and NuEra Tight specifically for the treatment of thigh cellulite. Consistent with previously reported data $[1-3,12]$, this non-invasive treatment modality does not lead to total resolution of the presenting skin contour deformity. However, given the minimal downtime and high patient acceptance, the moderate improvement offered by this technology leads to high patient satisfaction overall.

The current investigation does have several limitations. Most notably the sample size of 9 patients does not allow for rigorous statistical analysis of the results and incurs sampling bias. We were also not able to control for weight loss/gain during the study time frame. We believe our follow up duration of one month after the last treatment is sufficient to demonstrate long 
term efficacy, as we are not aware of a mechanism for cellulite recurrence, but longer-term follow up would be necessary to prove this.

Further studies will be needed to more precisely quantify the amount of improvement over a larger number of patients, but the current experience shows that the NuEra Tight RF system possess all the key characteristics needed for wide patient acceptance in the population seeking non-surgical treatment for cellulite.

In conclusion, we present a pilot study in the use of NuEra Tight for the treatment of thigh and buttock cellulite. Our results show both subjective and objective improvement, with a high ease of use rating by the providers and a high comfort rating by the patients.

\section{Acknowledgments}

This study was funded by a grant from Lumenis, Ltd. The role of the grant was for provider time compensation to carry out the study treatments and photography.

\section{Conflicts of interest}

This study was funded by a grant to the senior author (Dr. Clark Schierle) from Lumenis, Ltd.

\section{References}

1. Davis DS, Boen M, Fabi SG. Cellulite: patient selection and combination treatments for optimal results-a review and our experience. Dermatol Surg 2019;45:1171-84.

2. Hexsel D, Mazzuco R. Cellulite. In: Tosti A, Hexsel D, editors. Update in cosmetic dermatology. Berlin: Springer; 2013. p. 2132.

3. Callaghan DJ III, Robinson DM, Kaminer MS. Updates in cellulite reduction. Adv Cosmet Surg 2018;1:45-53.

4. Sadick N. Treatment for cellulite. Int J Womens Dermatol 2018;5:68-72.

5. Hexsel D, Fabi SG, Sattler G, Bartsch R, Butterwick K, Casab- ona $\mathrm{G}$, et al. Validated assessment scales for cellulite dimples on the buttocks and thighs in female patients. Dermatol Surg 2019;45 Suppl 1:S2-11.

6. Weiss ET, Barzilai O, Brightman L, Chapas A, Hale E, Karen J, et al. Three-dimensional surface imaging for clinical trials: improved precision and reproducibility in circumference measurements of thighs and abdomens. Lasers Surg Med 2009;41:767-73.

7. The American Society for Aesthetic Plastic Surgery. Cosmetic (aesthetic) surgery national data bank statistics [Internet]. Garden Grove (CA): The American Society for Aesthetic Plastic Surgery; c2018 [cited 2019 Jun 20]. Available from: https:// www.surgery.org/sites/default/files/ASAPS-Stats2018_0.pdf.

8. Hsu TS, Kaminer MS. The use of nonablative radiofrequency technology to tighten the lower face and neck. Semin Cutan Med Surg 2003;22:115-23.

9. Araújo AR, Soares VP, Silva FS, Moreira Tda S. Radiofrequency for the treatment of skin laxity: mith or truth. An Bras Dermatol 2015;90:707-21.

10. Nassab R. The evidence behind noninvasive body contouring devices. Aesthet Surg J 2015;35:279-93.

11. Wanitphakdeedecha $R$, Iamphonrat $T$, Thanomkitti K, Lektrakul N, Manuskiatti W. Treatment of abdominal cellulite and circumference reduction with radiofrequency and dynamic muscle activation. J Cosmet Laser Ther 2015;17:246-51.

12. Emilia del Pino M, Rosado RH, Azuela A, Graciela Guzmán M, Argüelles D, Rodríguez C, et al. Effect of controlled volumetric tissue heating with radiofrequency on cellulite and the subcutaneous tissue of the buttocks and thighs. J Drugs Dermatol 2006;5:714-22.

13. Manuskiatti W, Wachirakaphan C, Lektrakul N, Varothai S. Circumference reduction and cellulite treatment with a TriPollar radiofrequency device: a pilot study. J Eur Acad Dermatol Venereol 2009;23:820-7.

14. Fitzpatrick R, Geronemus R, Goldberg D, Kaminer M, Kilmer S, Ruiz-Esparza J. Multicenter study of noninvasive radiofrequency for periorbital tissue tightening. Lasers Surg Med 2003;33:232-42. 